\section{John Locke, philosophe qui ne voulait pas « se mêler de médecine »...}

\author{
Claire Crignon
}

Dans une lettre adressée en 1693 à son ami médecin Thomas Molyneux (1661-1733), membre du collège royal des médecins irlandais, John Locke (1624-1704), philosophe anglais du xvII siècle, auteur de l'Essai sur l'entendement humain mais aussi du Second traité du gouvernement civil et de la Lettre sur la tolérance (1689) déclare la chose suivante: "Si mon zèle à sauver les vies des hommes et à préserver leur santé (chose qui doit être infiniment préférée à toute spéculation médicale, aussi raffinée soit-elle) m’a emmené trop loin, veuillez l'excuser chez quelqu'un qui souhaite à la pratique médicale de réussir bien qu'il ne s'en mêle pas » [1].

Cette déclaration peut surprendre. Pour deux raisons. Si on la lit tout d'abord au prisme de la longue histoire des échanges entre médecine et philosophie, on rappellera l'existence d'une longue tradition de «médecins-philosophes » ou de «philosophes-médecins », qui remonte à Galien, passe par Avicenne, Cabanis, Jaspers, pour aller jusqu'à Anne Fagot-Largeault ou Anne-Marie Moulin ${ }^{1}$. Cette tradition suggère que la médecine pourrait constituer l'aboutissement de la philosophie dite «naturelle», celle qui s'occupe de rendre compte des phénomènes naturels et de leurs causes [2]. Or Locke a consacré de nombreux écrits à la philosophie naturelle, et c'est même dans ce domaine, qui inclut la médecine, mais aussi ce que nous appelons aujourd'hui biologie, chimie, botanique, physique et cosmologie, qu'il a commencé à se former et à écrire [3]. Pourtant, ce n'est pas dans cette lignée de «philosophes-médecins » qu'il souhaite s'inscrire. Sa position est, sur ce point, semblable à celle du médecin Thomas Sydenham, avec lequel il collabore étroitement et qui déclare, dans sa Méthode complète pour guérir presque toutes les maladies, qu'aucun patient ne peut raisonnablement souhaiter avoir un Descartes comme médecin: «II vaut mieux, pour l'utilité commune, être Médecin que Philosophe, car qui voudrait avoir Descartes pour son médecin ? » [4]. On sait par ailleurs que l'auteur de l'Essai sur l'entendement humain (1690) a pratiqué la médecine. Sa correspondance porte la trace des nombreuses sollicitations qui lui sont adressées, tout au long de sa vie, pour soulager des douleurs récalcitrantes, comme les maux de tête.

Vignette (Arthur Coga's blood transfusion, 1667 - Public domain review)

${ }^{1}$ Galien, médecin romain du $\|^{e}$ siècle après JC. Avicenne, médecin et philosophe persan des $X^{e}$ et $\mathrm{XI}^{e}$ siècles. Pierre-Jean-Georges Cabanis, médecin français de la seconde partie du XVIII ${ }^{e}$ siècle. Karl Jaspers, psychiatre et philosophe allemand mort en 1969. Anne Fagot-Largeaut et Anne-Marie Moulin ont toutes deux exercé la médecine tout en accomplissant leur carrière de chercheuses dans les champs de la philosophie de la médecine et de la bioéthique.

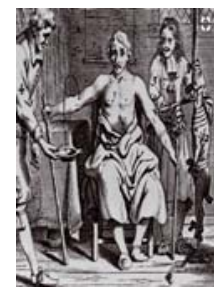

Université Paris-Sorbonne,

UFR de philosophie et sociologie,

1, rue Victor Cousin, 75005, Paris, France.

claire.crignon@paris-sorbonne.fr

On peut être encore surpris si on lit la déclaration de Locke avec un regard plus contemporain. Aujourd'hui, on ne cesse de demander aux intellectuels et, particulièrement, aux philosophes de se prononcer sur les questions épineuses posées par l'évolution des pratiques médicales ou les découvertes réalisées dans le domaine de la recherche biomédicale. Les philosophes, même sans avoir suivi des études de biologie ni de médecine, sont particulièrement sollicités pour prendre position dans les débats dits de «bioéthique », pour éclairer les valeurs et les normes qui peuvent entrer en conflit, à l'occasion des prises de décision médicale [5]. Ils se mêlent aussi de médecine en interrogeant ses ambitions sur un plan épistémologique et ontologique. La «philosophie de la médecine » est aujourd'hui considérée, certes après bien des débats, comme un domaine à part entière à l'intérieur de la philosophie des sciences [6].

À quel type de rapport entre philosophie et médecine faitil référence dans cette lettre? Au moment où Locke écrit, la philosophie et la médecine ne se présentent pas comme des disciplines autonomes et indépendantes, correspondant à des professions distinctes comme elles le sont aujourd'hui. La corporation des médecins commence à peine à se professionnaliser et les philosophes sont encore souvent dans le même temps des savants, qui s'adonnent à la médecine, à la physique, à la chimie ou à la botanique. $\mathrm{Ni}$ la philosophie ni la médecine n'existent véritablement comme professions ${ }^{2}[7]$. Du côté des patients, l'accès aux traitements médicaux reste limité à une classe privilégiée

${ }^{2}$ Voir les travaux de C. Rabier à ce sujet ainsi que ceux de T. Gelfand [7]. 
de la société, qui a les moyens de disposer d'un médecin personnel, les autres ayant majoritairement recours à des praticiens beaucoup moins savants, de simples apothicaires, des sages-femmes, des barbiers, parfois même ceux qu'on appelle des «charlatans », qui promettent des remèdes miraculeux et font miroiter l'espoir d'une santé meilleure à l'aide d'élixirs de longue vie et autres panacées miraculeuses. Les évolutions de la science et des techniques médicales ne sont pas considérées comme un objet de réflexion requérant l'expertise du philosophe ni la mise en relation avec d'autres types de savoirs spécifiques, puisque ce type d'interrogation fait partie intégrante des traités rédigés par les médecins, qui s’interrogent eux-mêmes sur les limites de leur art et ne séparent pas la question des transformations du savoir de celle de ses implications pratiques (morales ou politiques).

Lorsque Locke déclare ne pas se mêler de médecine, mais souhaiter seulement à la pratique médicale de réussir, il n'entend pas pour autant écarter toute nécessité d'une réflexion morale relative aux objectifs poursuivis par le médecin. La visée d'une vie en bonne santé, que ce soit sur le plan somatique ou psychologique, est en effet la condition sine qua non de l'épanouissement des êtres humains dans leurs activités quotidiennes, mais elle engage aussi notre vie morale et notre existence sociale et politique. Cependant, ce n'est pas là une tâche réservée à la seule médecine: les parents et les éducateurs peuvent (et doivent) aussi s'en soucier. II écrit ainsi dans ses Quelques pensées sur l'éducation parues en 1693 : «Que la santé est nécessaire à nos affaires et à notre bonheur, et que pour faire quelque figure dans le monde, nous ne pouvons nous passer d'un tempérament vigoureux, qui résiste au travail et à la fatigue : c'est un point évident, où la preuve est inutile. En parlant ici de la santé, mon dessein n'est pas de dire comment un médecin doit soigner un enfant malade ou débile : je veux seulement indiquer ce que, sans recourir à la médecine, les parents ont à faire pour conserver et développer chez leurs enfants une constitution saine ou tout au moins exempte de maladies » [8].

II ne faut donc pas confondre les rôles ni céder à la tentation de vouloir appliquer rétrospectivement à la pensée d'un auteur du xvII ${ }^{\mathrm{e}}$ siècle des notions ou un cadre de pensée apparus bien plus tard, dans un contexte tout à fait différent. Pour Locke, le philosophe n'est pas médecin, même s'il s'est formé à cette discipline et qu'il s'intéresse au rôle que joue la santé dans la visée d'une vie heureuse. II n'est pas un praticien en charge de diagnostiquer ni de définir des remèdes. Est-ce à dire que son rôle serait celui d'un théoricien fournissant au médecin les éléments qui lui feraient défaut pour sa pratique? C'est souvent de cette manière un peu simpliste que l'on a présenté le rôle joué par Locke auprès du médecin Thomas Sydenham (16241689), avec lequel il collabore régulièrement entre 1666 et $1671^{3}$.

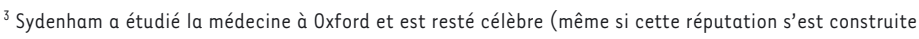
rétrospectivement) pour avoir appliqué à l'étude des maladies la méthode hippocratique de l'observation, tout en assignant aussi à la médecine une ambition systématique de classification des maladies. Son nom est associé à un certain nombre d'innovations thérapeutiques, en particulier dans le traitement des fièvres, mais aussi de la variole, qui fait alors de très importants dégâts parmi la population londonienne. Si ses méthodes de traitement ne font pas l'unanimité de son vivant, il est considéré comme une figure importante de la médecine moderne, et c'est à lui que l'on a longtemps attribué des manuscrits lockiens sur la médecine, inclus dans l'édition de ses œuvres complètes.
}

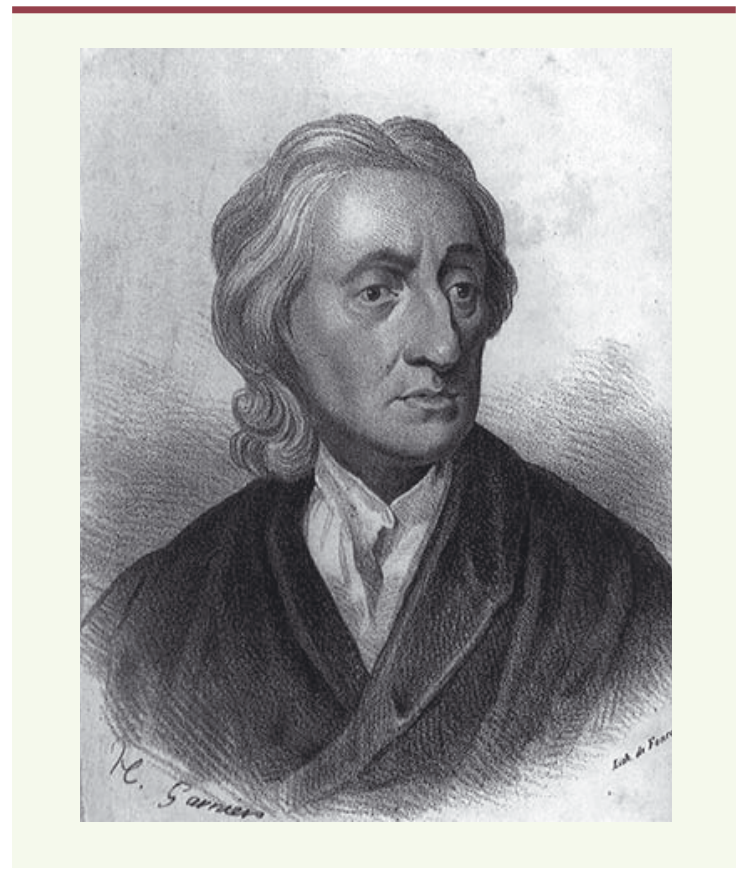

John Locke (1624-1704)

La remarque de Locke au sujet du danger d'une approche trop spéculative s'applique d'ailleurs aussi bien à la philosophie qu'à la médecine. Cette phrase n'indique pas un partage entre une discipline, la philosophie, qui serait par définition théorique et spéculative, et une autre, la médecine, qui serait quant à elle orientée vers la pratique et l'efficacité. Elle souligne bien plutôt un point commun entre médecine et philosophie : la nécessité pour le médecin, comme pour le philosophe, de se détourner d'une spéculation qui conduit le plus souvent à des conflits (entre sectes philosophiques ou médicales) ou à des controverses qui sont les principaux vecteurs d'un malaise ou d'une souffrance psychiques, qui font non seulement obstacle au progrès de nos connaissances, mais aussi à la visée d'une vie heureuse. Chez Locke, ce n'est pas tant la philosophie qui est sollicitée pour réfléchir sur la médecine, que la médecine qui sert à défendre une certaine conception de la philosophie, comme pratique, orientée vers la recherche du bien-être.

Pour le comprendre, il faut revenir sur les circonstances qui l'ont conduit à se former à ce domaine du savoir et à cette pratique. Étudiant à l'université d'Oxford, le jeune Locke obtient son Master of Arts le 29 juin 1658. La médecine fait alors partie, comme le droit, des disciplines permettant de se soustraire aux obligations ecclésiastiques et constituant, par ailleurs, une activité permettant de gagner sa vie. De 1659 à 1666, date de son départ pour Londres, Locke participe aux recherches expérimentales qui sont alors menées de manière très active par ses collègues médecins, physiciens ou 
chimistes. En effet, suite à la publication en 1628 du traité de William Harvey sur la circulation sanguine (De motu cordis), c'est l'ensemble des fonctions vitales (circulation, respiration, digestion, etc.) qu'il convient de repenser, à partir de principes qui viennent remettre en question l'héritage hippocratico-galénique et le rôle que jouent les humeurs dans la santé et la maladie. C'est aussi la thérapeutique qui doit être revue, avec les premières tentatives de transfusion sanguine, opérées d'abord sur les animaux avant d'être tentées sur l'être humain et rapidement abandonnées [9] ${ }^{4}$. On comprend donc que, dans ce contexte de profonde remise en question des savoirs antérieurs, l'état du savoir médical apparaisse extrêmement controversé.

Quelles sont les questions qui occupent plus particulièrement Locke au cours de ses années d'étude à 0xford ? L'étude des manuscrits rédigés entre 1666 et 1670, longtemps attribués au médecin Thomas Sydenham [10], mais dont on sait maintenant qu'ils ont été non seulement rédigés mais conçus par Locke (ou en étroite collaboration avec celui qu'on surnomme «l'Hippocrate anglais » [11]), apporte des réponses précises. On y voit Locke s'intéresser aux recherches sur le sang, à la respiration, au rôle de l'air dans certaines maladies professionnelles, à la genèse des maladies. Dans deux de ses textes, en particulier Respirationis Usus et Morbus (1666), l'étudiant en médecine rend compte de disputes virulentes qui opposent, en ce milieu du xvII ${ }^{e}$ siècle, les partisans de différents modèles du corps : ceux qui, suivant Hippocrate et Galien, se réfèrent à la théorie des quatre humeurs, les adeptes de la médecine alchimique et chimique, qui expliquent la genèse des maladies à partir de la présence de ferments et de principes dynamiques (comme «l'archée » ou principe de vie théorisé par le médecin flamand Van Helmont), et enfin les partisans d'une explication mécaniste des phénomènes naturels qui, à la suite de Galilée et de Descartes, s'efforcent d'appliquer au corps humain les principes d'une explication par « grandeurs, figures et mouvements » [12].

Comment Locke présente-t-il ces disputes? Le texte évoque une «guerre violente » opposant les partisans de la médecine chimique aux humoristes au sujet de la définition d'un principe thérapeutique, les premiers favorisant le principe d'une guérison par les semblables, quand les seconds défendent celui d'une guérison par les contraires. Les désaccords entre médecins sont présentés dans ce texte (et dans d'autres) comme de véritables disputes sectaires, qui, loin de contribuer à éclairer les questions posées, ne font qu'entretenir les doutes et nourrir des disputes interminables $[13]^{5}$. Lorsque Locke déclare, dans Anatomia, qu'il ne faut pas attendre des découvertes anatomiques un quelconque progrès pour l'art médical, il ne se prononce pas sur le contenu des avancées, indéniables dans ce domaine. II met simplement en garde contre l'illusion, qui guette les médecins, de croire qu'une plus grande visibilité du corps se traduira immédiatement par une plus grande intelligibilité du fonctionnement du corps [14]. II y a, en médecine, comme en religion, une tendance de l'esprit humain au dogmatisme, tendance que l'on ne peut pas reprocher aux hommes qui

\footnotetext{
${ }^{4}$ Voir aussi, pour une approche plus contemporaine sur la transfusion, les travaux de Jean Héritier, de Marie-Angèle Hermitte et d'Anne-Marie Moulin.

${ }^{5}$ Ce constat peut aussi s'appliquer à d'autres périodes. Voir par exemple [13] pour la médecine du XIXe siècle.
}

recherchent naturellement un «socle où asseoir leurs pensées » et «quelque fondement pour les guider dans la pratique de leur art», et qui ont par conséquent tendance à transformer de simples «hypothèses » en «principes sacrés » [15], qu'ils défendent avec la ferveur de sectateurs religieux, la recherche de la santé n'étant pas sans parenté avec celle du salut [16].

Comment aider alors les médecins à sortir de cet état permanent de conflit et de dispute ? Sans doute, en les incitant à revenir à la véritable finalité de l'art médical, finalité qui n'est pas, aux yeux de Locke, de parvenir à percer l'essence des maladies ni leurs causes cachées, mais de trouver des traitements qui permettront de «soulager les infirmités », de calmer la douleur et de contribuer au «bien-être » des malades [17]. Renoncer à l'ambition de donner à la médecine l'aspect formel d'une science, admettre qu'on ne dispose d'aucune connaissance certaine au sujet des causes, ce n'est pas renoncer à faire progresser la médecine, mais c'est, au contraire, se donner les moyens d'éviter les disputes interminables, en définissant une méthode susceptible d'aider le voyageur, qui traverse une épaisse forêt, à ne pas s'égarer parmi les bruyères et les aubépines: «Celui qui, en médecine, pose des maximes fondamentales pour en tirer des conséquences et donner lieu à des disputes, confèrera à celle-ci l'aspect formel d'une science. Il aura alors assurément fait quelque chose pour développer l'art de parler et peut-être posé le fondement de disputes sans fin. Mais s'il espère par un tel système, conduire les hommes à la connaissance des infirmités du corps, de la constitution, de la nature, des signes, des changements et de l'histoire des maladies, tout en indiquant une voie de guérison sûre et directe, il suit le même chemin que celui qui parcourrait en tout sens une épaisse forêt envahie de bruyères et d'aubépines, en pensant ainsi avoir une vue d'ensemble qui lui permette de dresser une carte du pays. Ces théorèmes spéculatifs sont aussi peu bénéfiques à la médecine qu'à l'alimentation humaine » [18].

En se formant à la médecine, Locke a pu mesurer les bénéfices d'un type de savoir qui poursuit d'abord l'objectif pratique d'améliorer le bien-être des humains avant toute ambition spéculative. En philosophie, comme en médecine, il convient non pas de commencer par chercher à mesurer l'étendue de tout ce que nous pourrions connaître dans l'absolu, mais de déterminer, à l'intérieur de bornes étroites, les choses qu'il nous serait avantageux de connaître au cours de notre existence terrestre. Dans le manuscrit de 1677 consacré aux études ( Of study»), Locke insiste sur cette finalité pratique de la connaissance: si nous cherchons à acquérir du savoir, c'est pour notre propre 
bénéfice et celui des autres [19]. C'est là une réflexion essentielle pour comprendre le choix de la «plain and historical method», méthode de l'histoire naturelle et de l'observation que Locke a d'abord pratiquée sur le corps avant de l'appliquer à l'esprit humain et à ses facultés [20]. Ainsi la médecine aide aussi à poser la question de la méthode en philosophie. Avant même de chercher à statuer sur la nature de notre entendement, il convient de se demander comment on va enquêter sur cet objet bien particulier [21], qui est à la fois ce que nous cherchons à connaître, et ce qui rend possible cette connaissance. Ainsi, au lieu de procéder à une «physique de l'âme » qui conduirait à une approche purement spéculative de l'esprit à même de statuer sur sa nature (matérielle ou immatérielle, pensante ou corporelle), on cherchera plutôt à observer et à décrire ses opérations, son mode de fonctionnement ou ses dysfonctionnements [22]. $\varepsilon t$ à cette « histoire naturelle » de l'entendement humain (observation), on adjoindra un «régime de l'esprit » [23], thérapie à même d'aider le sujet connaissant à conduire son entendement et à éviter les disputes et les doutes qui engendrent l'inquiétude, le malaise («uneasiness ») [24]. Qu'il s'agisse de l'esprit ou du corps, notre objectif est d'abord d'éviter la douleur et de rechercher un certain bien-être ou plaisir [25]. Or, de ce point de vue encore, la médecine contribue à identifier les doutes qui sont sources de troubles et de malaises et dont la philosophie cherche à nous sortir. En faisant de la médecine une matrice permettant de poser la question des limites de notre entendement, Locke s'inscrit dans une tradition sceptique qui, depuis la réflexion du philosophe Sextus Empiricus, voit dans l'incertitude et la suspension du jugement non pas des maladies, mais des remèdes qui peuvent nous aider à lutter contre une certaine tendance au dogmatisme qui est source de tourment [26].

$\varepsilon n$ même temps, le médecin est bien souvent, dans sa pratique, contraint de prendre des décisions, même en situation d'incertitude. Dès lors qu'il est question de vie ou de mort pour un patient qui s'en remet au savoir des médecins, il ne peut être question de suspendre le jugement, il faut décider d'un traitement sans avoir de certitude quant à son issue favorable ou défavorable. Autrement dit, il y a aussi dans les écrits de Locke, des éléments permettant d'envisager un certain «transport » de la philosophie dans la médecine ${ }^{6}$ [27] : se mêler de médecine pour le philosophe, ce n'est pas insuffler de la spéculation dans la pratique, mais c'est montrer comment certaines opérations intellectuelles (en particulier, l'histoire et l'observation, le recueil empirique des données sensibles et des signes) constituent des ressources qui permettent d'envisager le progrès de la médecine comme art, quand bien même son statut de science des causes des maladies peut être mis en question.

Depuis son traité paru en 1666 (Methodus Curandi Febres) jusqu'à ses Observationes Medicae de 1676, Thomas Sydenham préconise en particulier une observation scrupuleuse des signes et des symptômes de la maladie, contestant la prétention des médecins à accéder à l'essence des maladies et à leurs causes. L'épidémie de variole

${ }^{6}$ Pour un commentaire sur les origines hippocratiques de cette formule et pour un commentaire, voir $A$. Fagot-Largeault [28]. qui frappe l'Angleterre dans les années 1667-1668 (après l'épidémie de peste de 1666) conduit Sydenham et Locke à s'interroger sur les effets contre-productifs des méthodes de traitement en vigueur et sur la nécessité d'un changement de méthode, susceptible de faire espérer aux malades une guérison. Deux manuscrits témoignent de cette réflexion conjointe du médecin et du philosophe, Smallpox Epistle Dedicatory et Smallpox Preface (1670), esquisses d'un traité projeté qui ne verra jamais le jour, mais qui expriment une préoccupation nette pour la prise en compte de l'objectif pratique et thérapeutique de la médecine. Et cet objectif doit, selon Locke et Sydenham, toujours l'emporter sur l'ambition médicale personnelle, le désir de renommée et le goût pour les disputes stériles entre partisans de sectes médicales opposées.

Mais Locke ne s'est pas seulement adonné à l'observation des maladies épidémiques (ou aux effets pathogènes des conditions de travail des mineurs sur la respiration). Il a aussi suivi des patients particuliers. Chargé de récolter des eaux aux vertus thérapeutiques pour soigner un personnage politique de premier plan, Lord Ashley (le futur premier comte de Shaftesbury, chef de file du parti Whig qui jouera ensuite un rôle important dans le tournant politique de la réflexion du philosophe), Locke le suit en 1667 à Londres comme médecin. Il est amené à observer de près la maladie, qui se déclenche à partir de 1668 : un kyste hydatique qui se traduit par des douleurs récurrentes, une perte d'appétit, une couleur jaune de la peau qui conduit les médecins à évoquer le symptôme de la jaunisse. Tout en étudiant l'évolution de la maladie jour par jour depuis mai 1668 jusqu'au mois de novembre de la même année, Locke détaille les symptômes et signes sensibles du mal, décrit les remèdes prescrits et leurs effets (ou absence d'effets), la pose d'un drain et l'évacuation des vésicules contenues dans le foie, jusqu'à la guérison du patient. II donne aussi son avis sur le cas et sur la marche thérapeutique à suivre, aux côtés d'autres grands médecins célèbres de son temps (Francis Glisson, Thomas Sydenham) [28]. Ces textes modèrent la tonalité sceptique ou pessimiste des propos sur l'anatomie, comme connaissance spéculative incapable d'aider les médecins à progresser dans la cure des maladies. Le médecin «qui se tient aux côtés de l'anatomiste», celui qui se trouve au chevet du malade, comme Locke l'a été au moment de la maladie de Lord Ashley, peut, en relevant les changements sensibles qui affectent l'urine, le sang, la semence, les excrétions, suggérer des «indications sur le moment propice pour purger, saigner 
ou administrer un quelconque remède ${ }^{7}$ [29]. L'observation, qui s'appuie uniquement sur les signes perceptibles sans prétendre atteindre les causes «obscures 》 ou «cachées 》 qui échappent à notre perception, constitue une véritable modalité de connaissance, susceptible de fonder cette «science des signes» (ou sémiologie) que l'Essai présentera comme la troisième espèce des sciences, celle des «moyens par lesquels l'on peut acquérir la connaissance des choses et la communiquer aux autres » [30].

Loin d'adopter une position surplombante ni de prétendre jouer le rôle d'expert pour la pratique médicale, le philosophe a donc tout à gagner à s'engager dans la voie d'une formation médicale. Le gain de ce «détour » apparaît à plusieurs niveaux. D'abord il permet de s'opposer à une pratique purement scolaire et universitaire de la philosophie. Ensuite, il permet positivement de définir les finalités de la recherche de la vérité : chez Locke, comme chez Descartes, la médecine apparaît comme le modèle même d'une connaissance pratique, à même d'aider les hommes à lutter contre la douleur occasionnée par les maladies, mais aussi à chasser la mélancolie ou le malaise, qui guettent celui qui recherche la vérité avec trop d’ardeur. Enfin, la médecine propose d'autres dispositifs de connaissance, d'autres modalités d'accès au savoir que ceux que nous fournit la raison. L'observation des signes, l'histoire et le recueil des faits empiriques ne constituent pas des solutions par défaut mais offrent de véritables ressources dans un contexte marqué par l'incertitude.

Si l'on peut être déçu par l'ambition modeste que Locke semble assigner au philosophe vis-à-vis de la pratique médicale (ne lui souhaiter que de « réussir »), il y a là cependant une prise de position originale susceptible de nourrir la réflexion actuelle sur les modalités d'une rencontre entre «les humanités » et « la médecine».

John Locke, philosopher who did not want "to get involved in medicine" ...

\section{LIENS D'INTÉRÊT}

L'auteur déclare n'avoir aucun lien d'intérêt concernant les données publiées dans cet article.

\section{RéFÉRENCES}

1. John Locke à Thomas Molyneux, 20 janvier 1693. In : de Beer ES, ed. The Correspondence of John Locke, vol. IV. Oxford : Clarendon Press, 1979: 630.

2. Crignon C, Lefebvre D. La médecine entre art et science dans les traditions philosophiques et médicales de l'Antiquité à la période moderne. Paris : CNRS, 2018.

3. Anstey PA. John Locke and natural philosophy. Oxford : Oxford University Press, 2011.

4. Sydenham T. Méthode complète pour guérir presque toutes les maladies. In : Médecine pratique de Sydenham, trad. Jault AF. Paris : Barrois le Jeune, $1784: 561$.

${ }^{7}$ Une partie de ces observations est traduite dans Locke médecin. Manuscrits sur l'art médical [29].
5. Morizot B, Quivigier, Py. Noesis. Les limites de la bioéthique, $\mathrm{n}^{\circ} 28$. Paris : Vrin, 2016.

6. Giroux $\varepsilon$. «Philosophie de la médecine ». In : Barberousse A, Bonnay D, Cozic M, eds. Précis de philosophie des sciences. Paris : Vuibert, 2011 : 404-41.

7. Gelfand T. Professionalizing modern medicine. Paris surgeons and medical science and institutions in the $18^{\text {th }}$ century. Westport : Greenwood Press, 1980.

8. Locke J. Quelques pensées sur l'éducation, trad. Compayré G. Paris : Vrin, $1992 ;$ । (3-4) : 29.

9. Andrault R. «Guérir de la folie. La dispute sur la transfusion sanguine, 1667-1668 ». In : XVII siècle. Paris : PUF, 2014 : 509-32.

10. Dewhurst K. Dr Thomas Sydenham (1624-1689): his life and original writings. Londres: The Wellcome Medical Library, 1966.

11. Anstey PR. «The creation of the English Hippocrates ». In : Farnham, Dawson and Sons, eds. Medical history, 2011 ; 55 (4) : 457-78.

12. Descartes R. Principes, IV, 199. In : Adam et Tannery, eds. Fuvres de Descartes. Paris : Vrin, $1989: 317-8$.

13. Locke J. Respirationis Usus (1666), 7lv, trad. C. Crignon. In : Locke médecin. Paris : Garnier, $2016: 374$

14. Weisz G. The medical mandarins. The French Academy of medicine in the nineteenth and early twentieth centuries. Oxford: Oxford University Press, 1995.

15. Locke J. Anatomia, 32v. In : Locke médecin. Paris : Garnier, 2016 : 395-6.

16. Locke J. De Arte Medica (1669). In : Locke médecin. Paris : Garnier, 2016 : 410.

17. Locke J. Lettre sur la tolérance (1686). Spitz JF, ed. Paris : GarnierFlammarion, 1992 : 184.

18. Locke J. De Arte Medica, 49r. In : Locke médecin. Paris : Garnier, 2016 : 408.

19. Locke J. De Arte Medica, 49r. In : Locke médecin. Paris : Garnier, $2016: 411$.

20. Locke J. Of study. In : Axtell JL, ed. The Educational Writings of John Locke. Cambridge : Cambridge University Press, $1968: 407$.

21. Locke J. Essai sur l'entendement humain. Hamou P, ed. Avant-propos, trad. P. Coste. Paris : Livre de Poche, 2009 ; 2 : 126.

22. Locke J. Essai sur l'entendement humain. Hamou P, ed. Préface, trad. P. Coste. Paris : Livre de Poche, $2009 ; 2: 68-9$.

23. Hamou P. Dans la chambre obscure. Locke et l'invention du mind. Paris : Ithaque, 2018.

24. Corneanu S. Regimens of the mind, Boyle, Locke, and the early modern cultura animi tradition. Chicago : Chicago University Press, 2011.

25. Locke J. Essai sur l'entendement humain (livre II). In : Hamou P, ed. trad. P. Coste. Paris : Livre de Poche, 2009 ; xxi, §§ 31-33 : 412-4.

26. Locke J. Essai sur l'entendement humain (livre II). Des modes du plaisir et de la douleur. In : Hamou P, ed. trad. P. Coste. Paris : Livre de Poche, 2009 ; $x x$, p. 384-90.

27. Sextus Empiricus. Esquisses pyrrhoniennes, trad. P. Pellegrin. Paris : Seuil, 1997, livre I, 4 et 12, p. 59 et 69-73.

28. Fagot-Largeault, A. Leçon Inaugurale, jeudi $1^{\text {er }}$ mars 2001. Paris : Collège de France, 2001.

29. Locke J. De Arte Medica, In : Locke médecin. Manuscrits sur l'art médical. Observations sur le cas d'Anthony Ashley Cooper, 1668. Paris : Garnier, 2016 : 435-64.

30. Locke J. Anatomia, 34v. In : Locke médecin. Paris : Garnier, 2016 : 399-400.

31. Locke J. Essai IV, xxi, §l, p. 1036.

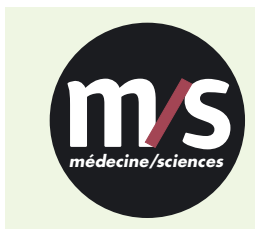

Tarifs d'abonnement $\mathrm{m} / \mathrm{s}-2018$

Abonnez-vous

à médecine/sciences
$>$ Grâce à $m / s$, vivez en direct les progrès des sciences biologiques et médicales

Bulletin d'abonnement page 98 dans ce numéro de $\mathrm{m} / \mathrm{s}$
TIRÉS À PART

C. Crignon 